\title{
Atlas of the open reading frames in human diseases: dark matter of the human genome
}

\begin{abstract}
Background: The human genome encodes RNA and protein coding sequences, the non-coding RNAs, pseudogenes and uncharacterized Open Reading Frames (ORFs). The dark matter of the human genome encompassing the uncharacterized proteins and the non-coding RNAs are least well studied. However, they offer novel druggable targets and biomarkers discovery for diverse diseases.
\end{abstract}

Methods: In this study, we have systematically dissected the dark matter of the human genome. Using diverse bioinformatics tools, an atlas of the dark matter of the genome was created. The ORFs were characterized for gene ontology, mRNA and protein expression, protein motif and domains and genome-wide association.

Results: An atlas of disease-related ORFs $(n=800)$ was generated. A complex landscape of involvement in multiple diseases was seen for these ORFs. Motif and domains analysis identified druggable targets and putative secreted biomarkers including enzymes, receptors and transporters as well as proteins with signal peptide sequence. About ten percent of the ORFs showed a correlation of gene expression at the mRNA and protein levels. Genome-Phenome association tools identified ORF's association with autoimmune, cardiovascular, cancer, diabetes, infection, metabolic, neurodegenerative and psychiatric disorders. Tumors encompassed largely heterozygous mutations.

Conclusions: The dark matter human proteome atlas reported in this study should aid in the discovery of novel therapeutic and diagnostic targets for multiple diseases
Volume 2 Issue I - 2015

\author{
Ana Paula Delgado, Maria Julia Chapado, \\ Pamela Brandao, Sheilin Hamid, Ramaswamy \\ Narayanan \\ Department of Biological Sciences, Charles E. Schmidt College \\ of Science, Florida Atlantic University, USA
}

Correspondence: Ramaswamy Narayanan, Department of Biological Sciences, Charles E. Schmidt College of Science, Florida Atlantic University, FL 3343 I, USA. Tel + |56 |2972247, Fax+| 56 |2973859, Email rnarayan@fau.edu

Received: January 08, 2015 | Published: January 30, 2015

Keywords: bioinformatics, biomarkers, body fluids, breast cancer, cancer proteome, dark matter, druggable targets, human genome, open reading frames, pancreatic cancer, prostate cancer, uncharacterized proteins

\begin{abstract}
Abbreviations: ClinVar, clinical variations; COSMIC, catalogue of somatic mutations in cancer; eQTL, expression quantitative trait loci; EBI-EMBL, european bioinformatics instituteeuropean molecular biology laboratory; GAD, genetic association database; GtEx, genotype-tissue nomenclature committee; HapMap, haploid map; HGNC, human genome nomenclature committee; HPRD, human protein reference database; HPA, human protein atlas; MOPED, model organism protein expression database; PheGenI, phenotype-genotype integrator; ORF, open reading frame; RefSeq, reference sequence; SNP, single nucleotide polymorphism; TCGA, cancer gene atlas; RP, retinitis pigmentosa; CRD, cone-rod dystrophy; AI, amelogenesis imperfecta; JBTS, joubert syndrome; FTD, frontotemporal dementia; ALS, amyotrophic lateral sclerosis; ORFs, open reading frames
\end{abstract}

\section{Introduction}

A vast amount of the human genome still remains uncharacterized. ${ }^{1-4}$ The uncharacterized part of the human genome offers an attractive potential for novel target discovery for diverse diseases. ${ }^{5}$ While known genes are easier to follow in the laboratory, a gap exists in our knowledge of the human genome due to the unknown function of the uncharacterized proteome. ${ }^{4}$ This gap, the dark matter of the human genome encompassing the non coding RNAs and uncharacterized protein coding sequences constitute over half of the known genes. New therapeutics and early diagnostic markers are urgently needed for major illnesses including, cancer, cardiac diseases, diabetes, infection, metabolic and mental disorders and neurodegenerative diseases. ${ }^{6}$

Reasoning that the uncharacterized proteins may offer new opportunities for therapeutic intervention, we have embarked on a systematic characterization of the Open Reading Frames (ORFs) present in the human genome..$^{7-11}$ The Genetic Association Database (GAD) provided a starting point to identify disease relevant ORFs of the dark Matter proteome. ${ }^{12}$ The ORF hits from the GAD were complemented with other disease-oriented databases including the MalaCards ${ }^{13}$ and the NextBio Meta analysis tool. From these analyses, 800 ORFs were identified relevant to diverse diseases and disorders. These ORFs were characterized using a streamlined approach we have recently established. ${ }^{10,11,14,15}$ Using phenome-Genome analysis, protein motif and domains, functional annotation and mRNA and protein expression analysis tools, a detailed knowledgebase of the dark matter ORFs was established. Classes of druggable proteins (enzymes, transporters, channel proteins and receptors) and secreted biomarkers were identified. Disease association to Cancer, diabetes Type I and Type II, Systemic Lupus, Rheumatoid Arthritis, Crohn's Disease, Leprosy, Hepatitis C, Attention Deficit Disorder, Alcoholism, Cardiac diseases, Erectile Dysfunction and for various genetic disorders was seen with the dark Matter ORFs. The database of novel ORF proteins should aid in therapeutic target discovery for cancer and other diseases. 


\section{Materials and methods}

\section{Genome analysis}

The genome analysis was performed using the Genetic Association Disease, GAD database, ${ }^{12,16}$ the University of California Santa Cruz, UCSC Genome Browser, ${ }^{17}$ the ensembl Genome Browser, ${ }^{18}$ the National Center for Biotechnology Information, NCBI Gene, NCBI Aceview, ${ }^{19}$ the Sanger Institute Catalogue Of Somatic Mutations In Cancer, COSMIC, ${ }^{20}$ the Integrated Drug Discovery platform, canSar v2, ${ }^{21} \mathrm{cBioPortal},{ }^{22}$ International Cancer Genome Consortium, ICGC, ${ }^{23}$ the Roche Cancer Genome Database, Mutome DB, ${ }^{24}$ Expression Quantitative loci browser, eQTL, ${ }^{25}$ Genotype -Tissue Expression Project, GTEx ${ }^{26}$ and the European Bioinformatics Institute- European Molecular Biology Laboratory, EBI-EMBL tools.

\section{Transcriptome analysis}

The transcriptome analysis of the uncharacterized proteins was undertaken using the NCBI-UniGene, SAGE Digital Anatomical Viewer, ${ }^{27}$ Cancer Genome Anatomy Project, CGAP, ${ }^{28}$ Oncomine microarray analysis tool, ${ }^{29}$ the Array express, ${ }^{30}$ The Gene Expression Omnibus, GEO and the Gene Indices from the Dana Farber Cancer Institute. ${ }^{31}$

\section{Proteome analysis}

The uncharacterized ORFs were analyzed for structural proteomics using the UniProt Knowledge base, UniProtKB, ${ }^{32}$ Swiss Expasy server, Protein Database, PDB, post translational modification sites at Expasy, ${ }^{33}$ PredictProtein, ${ }^{34}$ MEta Sever for Sequence Analysis, MESSA $^{35}$ and the I -TASSER server. ${ }^{36}$ The protein motifs and domains were analyzed using the NCBI Conserved domain database, CDD,${ }^{37}$ The PFAM ${ }^{38}$ ProDom, ${ }^{39}$ InterProscan $4,{ }^{40}$ HMMER,${ }^{41}$ Signal P server, ${ }^{42}$ and the Eukaryotic Linear Motif prediction, ELM. ${ }^{43}$ The protein expression analysis was performed using multiple tools including the Human Protein Atlas, HPA,${ }^{44}$ the Multi Omics Proteins Expression Database, MOPED ${ }^{45}$ the Human Protopedia Reference Database, HPRD, ${ }^{46}$ the Human Proteome Map ${ }^{47,48}$ and Proteomes database, proteomics $\mathrm{dB} .^{49}$

\section{Knowledge-based Data mining}

The knowledge databases used in the study included GeneCards, ${ }^{50}$ GeneAtlasm, ${ }^{51}$ NextBio meta analysis tool, ${ }^{52}$ The MalaCards, ${ }^{13}$ On line Mendelian Inheritance in Man, OMIM, Human Genome Nomenclature Committee, HGNC, ${ }^{36}$ Gene Ontology, amigo, ${ }^{53} \mathrm{NCBI}$ SNP database, the NCBI Phenome-Genome integrator, PheGenI, ${ }^{25}$ the Expression Quantitative Trait Browser, eQTL, ${ }^{26}$ the NCBI Clinical Variations database, ClinVar, ${ }^{54}$ Ensembl database, The Strings Interactome, ${ }^{55}$ BioGrid,${ }^{56}$ IntAct,${ }^{57}$ the KEGG pathway and the DAVID functional annotation tool. ${ }^{58}$

\section{Data analysis}

The entire database of GAD, Human Protein Atlas, HPA and UniGene was downloaded as comma separated value (csv) files and the Excel filtering tool was used to scan for the ORFs. Batch analysis of the ORF database was performed for canSar, the MOPED, the DAVID annotation tool, the Human Proteome Map, Proteomics dB, the HPRD, the PheGenI and the eQTL browser. The NextBio Meta Analysis was performed for the individual ORFs.

For the bioinformatics tools, the basic options were used as default. The PheGenI tool was used with P-values set at $<10-5$ and the R-Squared at 0.3 .
Big data verification was performed by verification using two independent experiments. Prior to using a bioinformatics tool, a series of control query sequences were tested to evaluate the predicted outcome of the results. ${ }^{10,11,15}$

\section{Results}

\section{Landscape Complexity of the Dark Matter of the Human Genome}

The current landscape of the human genome in the context of coding and non-coding sequences is shown in Figure 1. The dark matter of the human genome encompassing the non coding RNAs (ncRNAs), the uncharacterized proteins and Open Reading Frames (ORFs) currently account for over half of the HGNC approved protein coding sequences $(10,225 / 19,052)$. In attempt to establish disease relevance for the protein ORFs, multiple disease-oriented databases (Genetic Association database, The MalaCards from Gene Cards and the NextBio Meta analysis tool) were used. The GAD was enriched for positive genetic association evidence and 2,375 genetic polymorphisms associated with ORFs were identified. These ORFs were filtered for disease classes using three filters i) broad phenotypes, ii) disease classes and iii) Medical Subject Headings terms. The Dark matter ORFs-related polymorphisms were detected for diverse diseases.

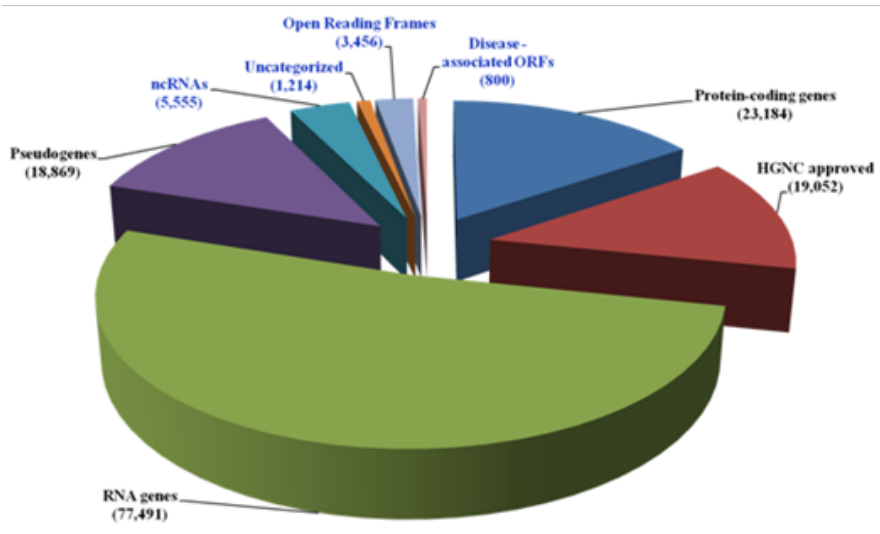

Figure I The human genome Dark Matter landscape.

The current number of genes (known and uncharacterized) was obtained from GeneCards, HGNC and UniProt. The disease associated ORFs were identified from the Genetic Association database, the MalaCards and the NextBio Meta analysis tool. The dark matter of the genome is shown in blue.

From these analyses, 800 ORFs were identified (Supplemental Table, S1). These ORFs termed as Dark Matter ORFs, were verified by batch analysis using the GeneALaCart tool from the GeneCards. From these studies a comprehensive knowledgebase (Dark Matter ORFs) was created (Supplemental Table, S2). Additional verification of the ORFs came from HGNC, UniProt and UniGene databases. This Dark Matter ORFs were classified into non coding RNAs $(n=47)$, uncharacterized $(\mathrm{n}=591)$ and known gene -related $(\mathrm{n}=164)$. Among these 800 ORFs, 415 ORFs were positive for genetic association evidence in the GAD.

Using advanced filtering option, the Dark Matter ORF database of genes was classified into broad classes of therapeutic areas (Figure 2). The number of ORFs for each therapeutic areas (blue bars) and the number of ORFs with genetically associated SNPs (red bars) is shown in Figure 2. Largest number of ORFs was associated with three major classes: infection $(\mathrm{n}=490)$, cancer $(\mathrm{n}=424)$ and metabolic disorder 
$(n=352)$. Association of the Dark Matter ORFs with multiple diseases encouraged us to undertake a further detailed analysis to provide an atlas of novel genes for target discovery.

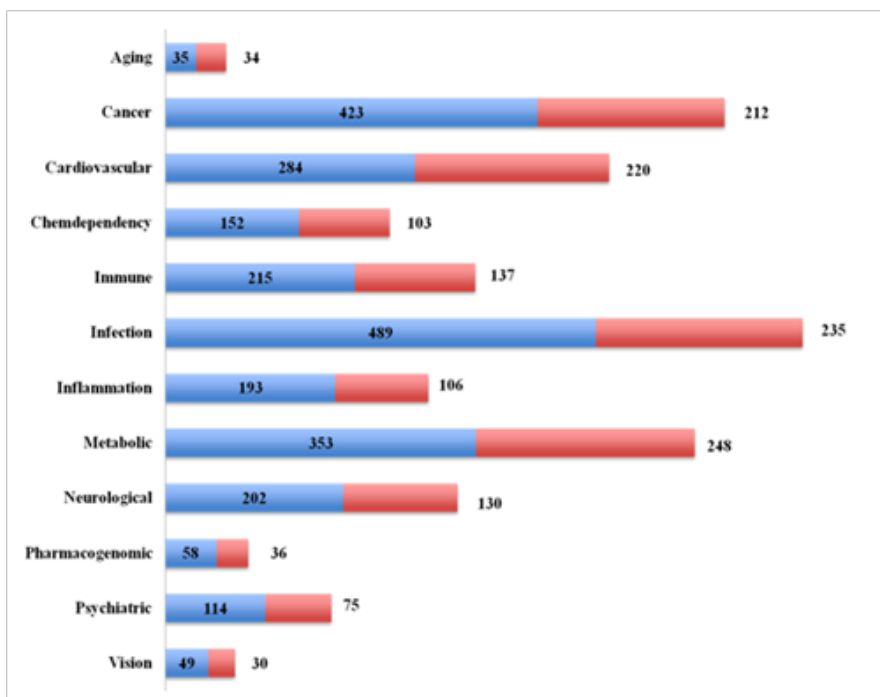

Figure 2 The Dark Matter ORFs in therapeutic areas.

The Dark Matter ORFs were identified from the Genetic Association Database (GAD), the MalaCards and the NextBio Meta Analysis tool. The disease-associated ORFs (protein coding and ncRNAs) shown in figure I were categorized into broad classes (blue) and genetically associated ORFs (red).

\section{Gene ontology}

The 800 Dark matter ORFs were batch analyzed for Gene Ontology (GO) using the DAVID functional Annotation tool. Additional GO verification was obtained from the GeneALacart, the GeneCards, the canSar and UniProtKB databases (Figure 3). The ORFs were classified according to the localization (panel A), function (panel B) and process (panel C). Druggable targets (enzymes, $\mathrm{n}=92$, integral membrane, $\mathrm{n}=237$, transporters, $\mathrm{n}=19$, receptors, $\mathrm{n}=6$ ) and putative secreted ORFs (extracellular region, $\mathrm{n}=82$ ) were identified by the $\mathrm{GO}$ analyses. Thirty-one of these extracellular ORF proteins harbored classical signal peptide sequence at the $\mathrm{N}$-terminus. Further, the cell localization data (see panel A) indicated various sub-cellular compartments including the Cytoplasm, Nucleus, Golgi, Mitochondria and Vesicles. The ORFs were classified into distinct binding classes including carbohydrate, lipid, metals, nucleotide and proteins (Panel B). The Dark Matter ORFs were also associated with diverse processes/pathways including apoptosis, cell cycle, differentiation, DNA repair, metabolic, signal transduction etc. (Panel C). These results provided additional insight into the nature and putative function of the Dark Matter ORFs and suggested their involvement in multiple cellular processes involving growth control and regulation.

\section{Gene expression}

The availability of numerous gene expression analysis tools for both mRNA and protein allowed us to establish the gene expression profile of the dark Matter ORFs in normal and diseased tissues. The mRNA expression in normal tissues was analyzed using the UniGene (Expressed Sequence Tag, EST) and the NextBio Meta Analysis tool (Microarray datasets). Additional verification of mRNA expression for normal and tumor tissues was derived from the Oncomine Microarray database. Multiple protein expression analysis tools (MOPED, HPRD, HPA, Human Proteome Map, and the Proteomics dB) were used to verify the protein ORF expression in diverse normal tissues including body fluids and tumor tissues (Supplemental Table, S3).
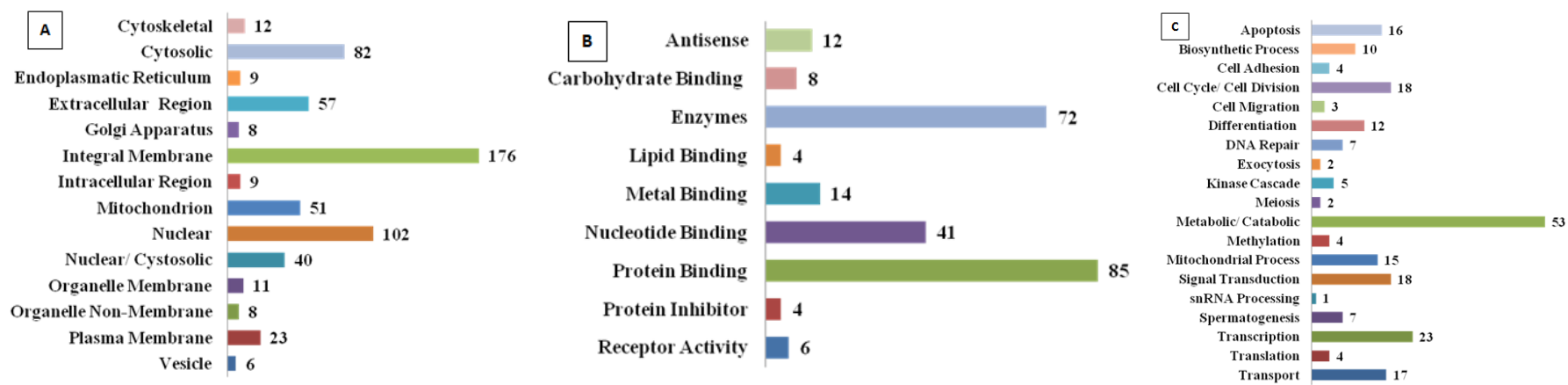

Figure 3 Gene Ontology of the Dark Matter ORFs.

Putative classes of the Dark Matter ORFs inferred from Gene Ontology tools (DAVID, GeneCards and UniProtKB). Panel A) localization; Panel B), function and Panel C) process.

ORF expression (mRNA and protein) was detected in diverse body fluids including blood, bone marrow, bronchoalveolar lavage, cerebrospinal fluid, cerumen, ascites, milk, proximal fluid, tear, saliva, semen, synovial fluid and urine ( $\mathrm{n}=102 / 800)$. A total of fiftyeight ORFs were found to have a classical signal peptide sequence at the N-terminus; eleven of these signal peptide-containing ORFs were detected in the body fluids. Further, tissue- and developmentally regulated expression was seen for diverse ORFs (Figure 4). In the adult, largest number of ORFs expression was seen in the testis $(n=319)$ followed by the brain $(n=135)$. Fetal, infant and adultrestricted expression was also seen for the ORFs. A correlation of expression at the mRNA and protein level across multiple datasets was seen in approximately $10 \%$ of the ORFs (87/800).

An ORF, C19orf10 interleukin 27 working designation, ${ }^{59,60}$ present in the pancreatic juice, bronchoalveolar lavage, milk, semen and urine was associated with pancreatic, prostate and hepatic carcinomas. This ORF is also associated with other diseases including Asthma, Cardiac transplant rejection, Pancreatitis, Encephalomyelitis, HIV-1, Genetic Predisposition to Inflammatory Bowel Disease and Crohn's disease. ${ }^{61}$ This novel putative cytokine ORF has potential for diagnostic marker development. Other ORFs present in the pancreatic juice included c19orf173, C14orf10, C5orf52, C12orf56 and C19orf43 and may offer target potential for pancreatic diseases including cancer.

The urinary ORFs included C11 orf52, C17orf37, C19orf10, C2orf54 and C9orf142. The C17orf37, a selenoprotein, located at the ERBB2 locus is associated with Breast cancer susceptibility ${ }^{62,63}$ and may provide a non-invasive urinary detection. The milk ORFs encompassed C6orf15, C11orf52, C11 orf67, C16orf178, C19orf10 and $\mathrm{C} 21$ orf23. 


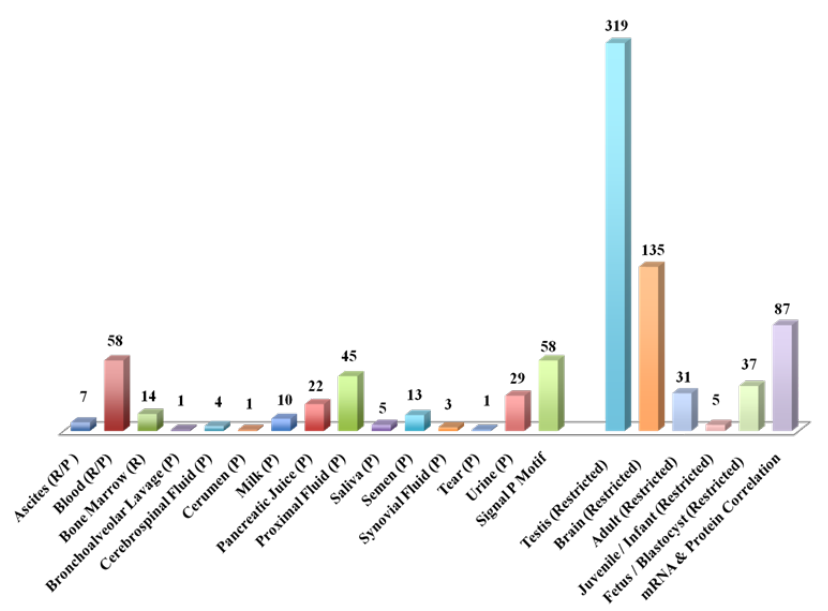

Figure 4 Gene expression analysis of the Dark Matter ORFs.

The mRNA and protein expression of the Dark Matter ORFs analyzed from the gene expression databases (UniGene, NextBio, MOPED, HPRD, HPA, human proteome map and proteomes $\mathrm{Db}$ ) is shown. The number of ORFs identified for each class is shown in top of the bar. Body fluids with mRNA (R) and protein $(\mathrm{P})$ level expression evidence are shown.

Further, the detection of the Dark Matter ORFs in other body fluids including the proximal and synovial fluids, semen and cerumen opens a new potential for minimally invasive and noninvasive diagnosis.

In addition to protein coding ORFs, ncRNAs were also detected in the body fluids. This included bone marrow (C21orf82, C21orf93,C21orf94, C21orf96) and tear (C9orf27).

The presence of protein coding ORFs and the ncRNAs in diverse body fluids offers a rationale for non-invasive methods of detection in multiple therapeutic areas.

\section{Phenome-genome analysis of the dark matter ORFs}

The Phenotype-Genotype Integrator (PheGenI), a phenotypeoriented resource facilitates follow-up studies from GWAS and allows prioritization of variants. An expression quantitative trait locus (eQTL) represents a gene marker (locus) in which variation between individuals is associated with a quantitative mRNA gene expression trait. ${ }^{64}$ The eQTL results encompass 1) a SNP marker; 2) the gene expression levels; and 3) a measure of the statistical association between the two in a study population, such as the P-value.

The Phenotype-Genotype Integrator (PheGenI) was used to establish phenotypic traits associated with the ORFs. Genetically associated traits were seen for 227/800 ORFs in diverse therapeutic areas (Figure 5). We next performed eQTL analysis for the phenotype positive ORFs. The ORFs were further analyzed using the eQTL browser for eQTL traits. Genotypes for the OncoORFs were selected for exons, introns, near gene and Untranslated Region (UTR). From the output of results, disease traits were enriched (Supplemental Table, S4).

Twenty-nine ORFs were identified for eQTL traits in major diseases and disorders including

i. cancer: C3orf21, C6orf204,C2orf43, C18orf34 (lung, renal, prostate),

ii. Diabetes Type I: C6orf27;

iii. Diabetes Type II: C6orf57, C1 orf131; iv. Systemic Lupus: C8orf13;

v. Rheumatoid Arthritis: C8orf13;

vi. Leprosy, Hepatitis C: C7orf44, C20orf194,

vii. Crohn's Disease: C11orf10;

viii. Alcoholism: C3orf59;

ix. Erectile Dysfunction: C9orf3;

x. Cirrhosis: C3orf11;

xi. Cardiovascular:C6orf204,C2orf43, C14orf118, C10orf107,

xii. Asthma: C13orf35 and

xiii. Attention Deficit Disorders: C12orf28.

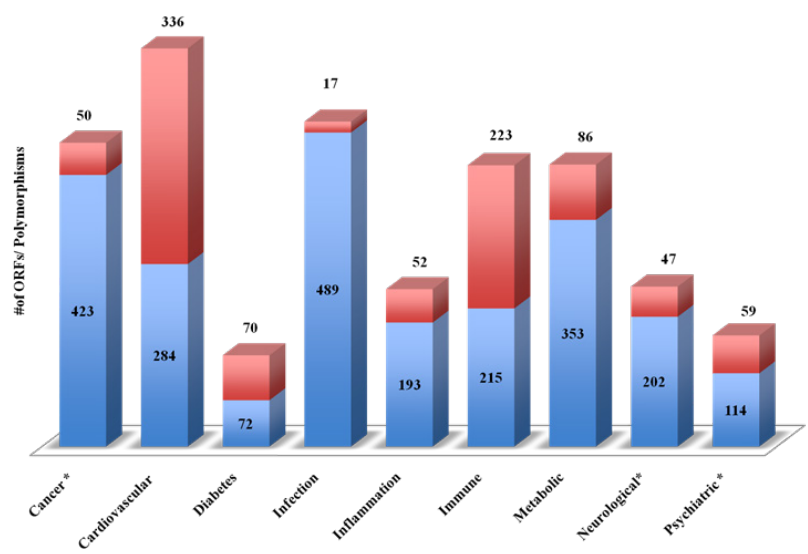

Figure 5 Genetic association of the Dark Matter ORFs in diverse diseases and disorders.

The Genetic Association Database (GAD) was enriched for genetic association and the number of polymorphisms associated with major therapeutic areas is shown (Red). The number of Landscape ORFs for each class is shown in blue. Clinical variation evidence indicated by *

Association with diverse traits was seen for some of the ORFs: C2orf43 (prostate cancer, Cholesterol, Echocardiography, Aspartate Aminotransferases); C11orf10 (Crohn's Disease, Cholesterol, Phospholipids, Lipoproteins); C8orf13 (Systemic Lupus, Rheumatoid Arthritis) and C6orf204 (Renal Carcinoma, Electrocardiography).

On the other hand, unique association was also seen with some of the ORFs in Non Small Cell lung carcinoma (C3orf21); prostate neoplasm (C18orf34); Leprosy (C7orf44); Chronic Hepatitis C (C20orf194); Blood pressure (C14orf118); Alcoholism (C3orf59); Attention Deficit Disorder, (C12orf28); Psychomotor Performance (C11orf73) and Erectile Dysfunction (C9orf3).

\section{Clinical variations}

We next established the clinical relevance of the ORFs using the NCBI Clinical variations database, ClinVar ${ }^{54}$ for pathogenicity and presence in clinical samples. Twenty-one ORFs were identified with clinical correlation and pathogenicity (Supplemental Table, S5).

Clinical significance was seen in developmental delay (C2orf43, C2orf48, C2orf50, C2orf61, C2orf73, C2orf91, C5orf42, C15orf41, C17orf105); Retinitis pigmentosa (C2orf71, C8orf37); Melanoma (C2orf16, C5orf42, C12orf57, C17orf104) and non small cell 
lung cancer (C10orf11, C17orf31); Oculocutaneous albinism (C10orf11); Recessive hypo mineralized amelogenesis imperfect (C4orf26); Amyotrophic lateral sclerosis and/or frontotemporal dementia (C9orf72); Temtamy syndrome (C12orf57); Spastic paraplegia 55, autosomal recessive (C12orf65); Chiari malformation type II (C17orf105); Autosomal dominant progressive external ophthalmoplegia (C10orf2); Spastic paraplegia 55, autosomal recessive (C12orf65); Anemia, Congential Dyserthropoitic (C15orf41) and Neurodegeneration with brain iron accumulation 4 (C19orf12). Dark Matter ORFs in multiple diseases.

In an attempt to develop a therapeutic area -related ORFs for druggableness and diagnostic markers identification, we next investigated the Dark Matter database for SNPs associated with major diseases. Figure 5 shows the number of dark matter ORFs and the SNPs associated with major therapeutic areas (cancer, cardiovascular, diabetes, infections, inflammation, immune, metabolic, neurological and psychiatry). About half of the ORFs were associated with infectious diseases and cancer ( $\mathrm{n}=490,424 / 800$ respectively). Cardiovascular and immune disorders were associated with the largest number of SNPs ( $\mathrm{n}=338$ and 225 respectively). Clinically relevant SNPs were identified using the NCBI ClinVar and were found in cancer, neurological and psychiatric illnesses.

Figure 6 shows the complex landscape of the dark matter ORFs across multiple diseases. A considerable overlap with multiple diseases was seen for most of the ORFs in the database. About $6 \%$ (56/800) of the ORFs showed a high degree of selectivity to distinct therapeutic areas. For example, enrichment was seen for cancer (C7orf71), infections (C1 orf 185, C1orf198, C7orf70, C9orf114, C11orf84, C19orf47), immune (C6orf100, C10orf64), inflammation (CXorf22) and neurological disorders (C20orf7).

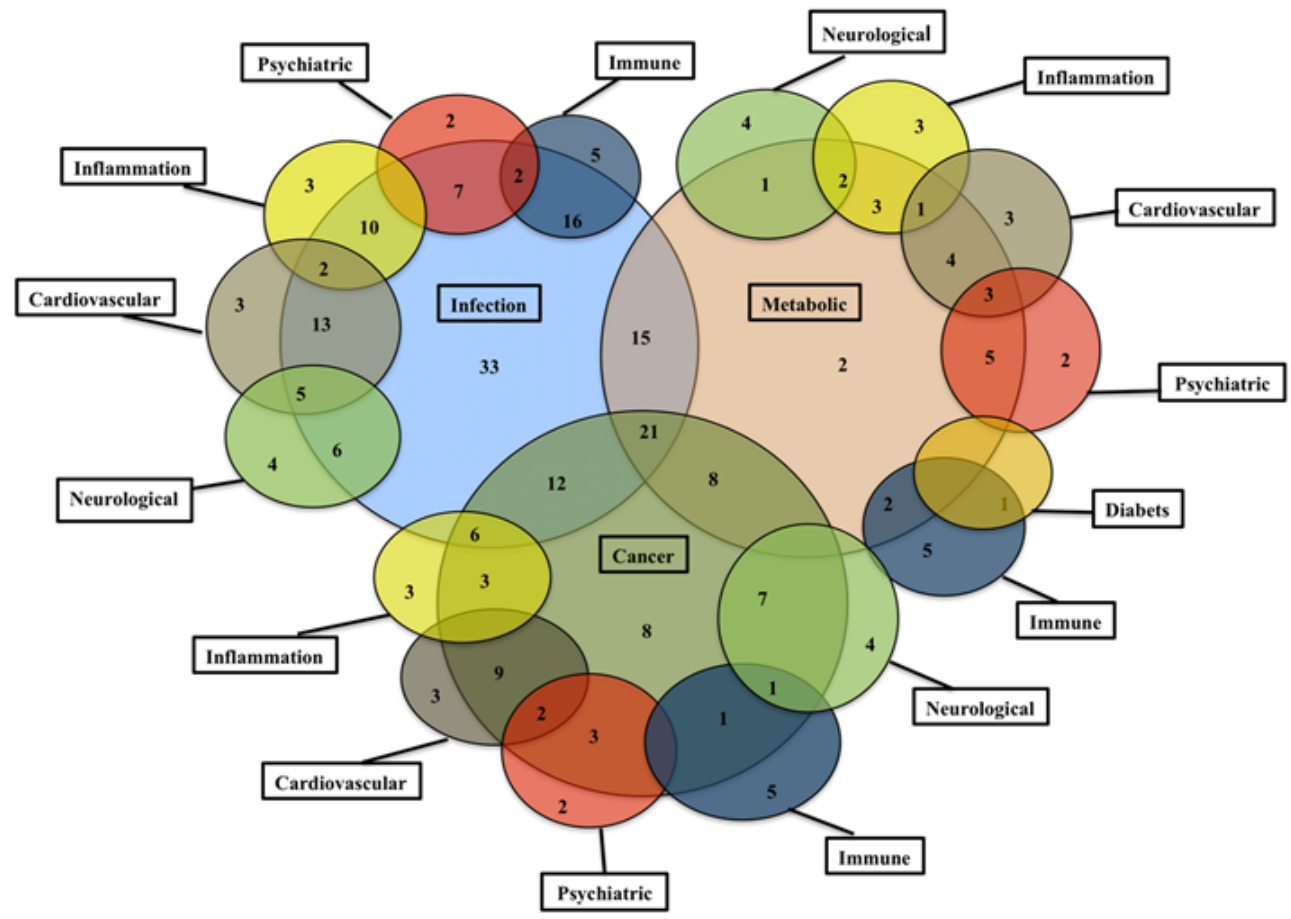

Figure 6 Dark Matter ORFs landscape across diverse diseases.

The Dark Matter ORFs were clustered using Excel clustering tool into individual therapeutic areas as shown and the ORFs shared across diverse diseases are shown. The numbers indicate ORFs shared across diseases (overlapping circle).

The C7orf71, a testis-enriched novel ORF showed strong association with neuroendocrine, brain, gastric tumors, lymphomas and HIV-1. ${ }^{65}$ The C19orf47, an Influenza viral infection-associated ORF, is a putative secreted protein with signal peptide sequence at the n-terminus and is present in blood plasma. This ORF offers a less invasive diagnostic potential. The C6orf100 is associated with Graves' disease, Systemic Lupus and Autoimmune endocrine diseases. ${ }^{66,67}$ The C10orf64, a WD repeat- and FYVE domain-containing protein 4 is associated with Systemic lupus, Autoimmune skin disease, Rheumatoid arthritis and Chemdependancy. ${ }^{66,68}$ The C20orf7, a probable mitochondrial methyltransferase| NADH dehydrogenase [ubiquinone] 1 alpha sub complex assembly factor 5, is associated with Paralytic syndrome, Mitochondrial Complex I Deficiency, Chronic fatigue syndrome and Chemdependancy. ${ }^{69-71}$

These results underscore the overlapping involvement of the ORFs in multiple diseases and emphasized the ORF landscape complexity.

\section{Cancer mutations of the dark matter ORFs}

The Catalogue of somatic mutations in cancer database, COSMIC has comprehensive mutation data for both the known and uncharacterized proteins. ${ }^{17}$ The entire COSMIC database was downloaded and enriched for the dark matter ORFs (Supplemental Table, S6).

The ORFs harbored diverse mutations (nonsense, missense, deletions, insertions, frame shifts, in-frames, homozygous and heterozygous) as shown in Figure 7. Heterozygous mutations accounted for the largest number of mutations (81.57\%) followed by substitution missense mutations (12.5\%). Major COSMIC mutations were present in Breast $(n=1,874)$, endometrium $(n=6,528)$, kidney $(\mathrm{n}=1192)$, Large Intestine $(\mathrm{n}=7,962)$, liver $(1,225)$, lung $(\mathrm{n}=7,445)$, esophagus $(n=813)$, Ovary $(n=833)$, pancreas $(n=323)$, prostate $(\mathrm{n}=569)$, skin $(\mathrm{n}=1,342)$, stomach $(\mathrm{n}=340)$ and urinary track $(\mathrm{n}=861)$. 


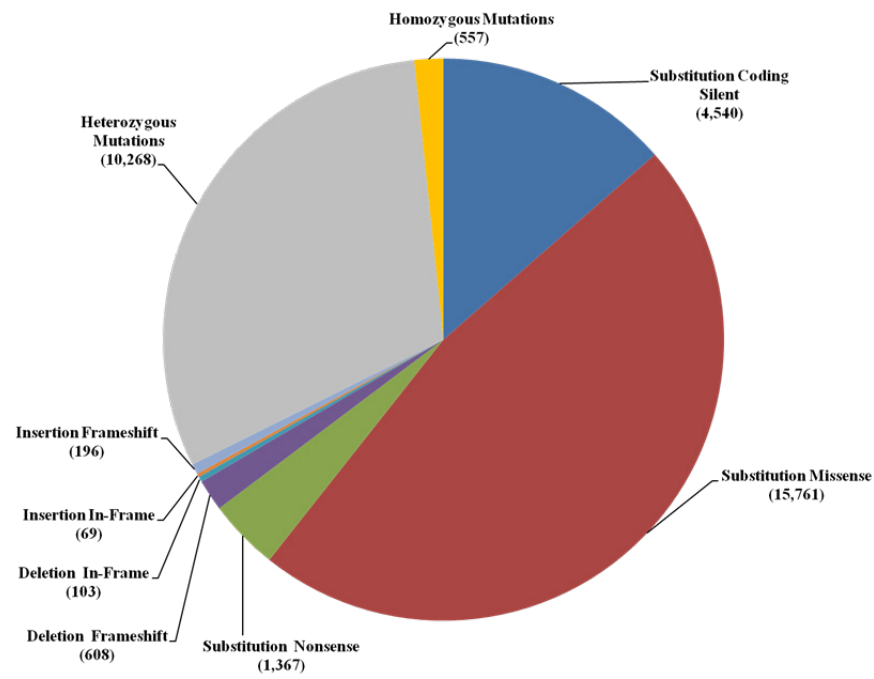

Figure 7 COSMIC mutational class of the dark Matter ORFs.

The mutations subtypes from the Catalogue of Somatic Mutations in Cancer (COSMIC) database are shown for the Dark Matter ORFs. Number of mutations for each subtype is shown in parentheses.

\section{Motif and domains analysis}

To develop further insight into the nature of the Dark matter proteins, the ORFs were analyzed for protein motif and domains. The GeneALaCart tool was used to batch analyze the ORFs for the InterPro/UniProt Domains and Families. ${ }^{72}$ In addition, the NCBI Conserved Domain Database, CDD, ${ }^{37}$ the Protein Family, PFAM ${ }^{38,73}$ the Biosequence analysis using profile hidden Markov models, HMMER,${ }^{41}$ the Protein Domain Analysis, ProDom, ${ }^{39}$ UCSC Genome Browser $^{74}$ and SignalP ${ }^{42}$ bioinformatics tools were used to analyze the Dark Matter ORFs. The post-translational modification sites, binary interactions and protein architecture and complexes data were obtained from the HPRD database batch analysis (Supplemental Table, S7).

\section{Noncoding RNAs in diseases}

Forty-seven ncRNAs were identified among the Dark Matter ORF database. These encompassed long intergenic RNAs, Linc RNAs $(\mathrm{n}=23)$, antisense transcripts/Linc RNAs $(\mathrm{n}=31)$ and pseudogenes $(n=4)$.These ncRNAs showed genetic association with diverse diseases including cancer, cardiovascular, diabetes, infections, inflammation, metabolic, neurological and psychiatric illnesses. Distinct ncRNAs were associated with type I diabetes (C6orf208) and type 2 (C6orf27, C14orf70). The ORF, C1 orf133|SERTA domain containing 4 antisense RNA 1, a putative uncharacterized protein showed association with celiac disease. The C18orf16|Aquaporin Isoform Delta 4 Antisense RNA was associated with both type 1 and type 2 diabetes. The C9orf29| leucine rich repeat containing 37 , member A5, pseudogene showed association with bladder and prostate carcinomas, multiple myeloma and plasmacytoma, Irritable bowel syndrome and Cytomegalovirus infection. The C14orf55 ADAM metallopeptidase domain 20 pseudogene 1 was associated with Multiple myeloma/plasmacytoma, Cancer of head and neck, Prostate cancer and Coronary arteriosclerosis. The C20orf191|nuclear receptor corepressor 1 pseudogene 1 was associated with Systemic lupus erythematous, Systemic inflammatory response syndrome, Lipoproteins, HDL and Uveitis. These results provide novel ncRNA targets for diverse diseases.
From these analyses, the Dark Matter ORFs were annotated into functional classes of proteins (Table 1). Protein families including antigens, carrier proteins, enzymes, nucleotide/metal binding, receptors, mitochondrial chaperones, phosphoproteins, secreted glycoproteins, selenoproteins, transporter/sorting proteins, vacuolar proteins and Zinc finger proteins were identified among the Dark matter ORF proteins.

Table I Protein characterization of the Dark Matter ORFs: Motifs and Domains

\begin{tabular}{ll}
\hline Protein family & Number of dark matter ORFs \\
\hline Antigen & 8 \\
Antisense & 35 \\
Carbohydrate/heparin binding & 6 \\
Channel & 4 \\
Enzymes & 118 \\
Glycoproteins & 41 \\
Metal binding & 24 \\
Nucleotide binding & 55 \\
Phosphoprotein & 18 \\
Protein Binding & 52 \\
Receptors & 20 \\
Secreted & 57 \\
Transcription factors & 32 \\
Transmembrane & 122 \\
Transporters & 9 \\
Vesicular & 1 \\
\hline
\end{tabular}

\section{Discussion}

We have embarked on systematically deciphering the uncharacterized ORFs, the Dark Matter proteome in the human genome. These ORFs are the least analyzed putative proteins in the genome and continue to remain a mystery. Realizing a target potential for these novel proteins for druggableness and diagnostic markers development, a comprehensive analysis of the ORFs was undertaken. Omics characterization was performed for 800 disease-oriented ORFs in the human genome. An atlas of the Dark Matter proteome was generated encompassing details on the somatic mutations, baseline mRNA and protein expression, protein motif and domains, disease traits and clinical relevance. Over half of the ORFs (416/800), analyzed in the study showed positive genetic association with diverse diseases and disorders. The disease landscape complexity of the ORFs offers an advantage to develop a single ORF target for novel therapy and diagnosis for diverse diseases. Omics correlation.

In a recent study, Zhang et $a 1 .{ }^{75}$ performed an extensive proteogenomic characterization of colon and rectal tumors using The Cancer Gene Atlas (TCGA) samples. Their results indicate a lack of correlation of gene amplification, mRNA and protein expression for over two thirds of the samples analyzed. Only a small number of genes showed a strong correlation of gene expression at the mRNA versus protein levels. This is not surprising in view of the issues such as stability and post -translational modification and regulation of protein expression. ${ }^{76}$ Our results concur with these findings: 87/800 
ORFs (about 10\%) showed correlation of expression at the mRNA and protein levels. For druggable target prediction as well as pathway mapping, such a correlation can facilitate target prioritization. Our study predicts secreted proteins as measured by mRNA and protein expression in diverse body fluids (102/800 ORFs) including blood, cerebrospinal fluid, synovial fluid, plasma, serum, bone marrow, Bronchoalveolar lavage, ascites, milk, pancreatic juice, semen, saliva, tear and Urine. Signal peptide sequence was detected in 58/800 ORFs. The baseline expression of the Dark Matter ORFs in the body fluids provides rationale for further investigation into secreted biomarkers characterization.

\section{The Noncoding RNAs}

The ncRNA class encompasses diverse non coding RNAs such as the long intergenic RNAs, micro RNAs, Small nuclear ribonucleic acids, Small interfering RNAs, the antisense transcripts and the pseudogenes. The ncRNAs play key roles in the regulation of gene expression at the transcriptome and proteome levels. Long considered as the Dark matter of the genome, these ncRNAs are increasingly becoming important in diagnosis and therapy of diverse diseases. ${ }^{77,78}$ The forty-seven ncRNAs comprising linc RNAs, antisense RNAs and pseudogenes discovered in this study, offer new target potential for diverse diseases including autoimmune disease, cancer, cardiovascular and inflammation. Further, the detection of some of the ncRNAs in the body fluids such as the bone marrow and tear offers diagnostic potential.

\section{Genome to Phenome association}

Disease-associated phenotypes were seen by eQTL analysis for 29 ORFs in diverse diseases including autoimmune diseases, cardiovascular diseases, cancer, infections, inflammation, and psychiatric disorders. Association with multiple traits was seen for some of the ORFs, for example, $\mathrm{C} 2$ or $\mathrm{f} 43$ (prostate cancer, Cholesterol, Echocardiography, Aspartate Aminotransferases); C11orf10 (Crohn's Disease, Cholesterol, Phospholipids, Lipoproteins); C8orf13 (Systemic Lupus, Rheumatoid Arthritis) and C6orf204 (Renal Carcinoma, Electrocardiography). On the other hand, unique phenotypic association was also seen with some of the ORFs such as in Non Small Cell lung carcinoma (C3orf21); prostate neoplasm (C18orf34); Leprosy (C7orf44); Chronic Hepatitis C (C20orf194); Blood pressure (C14orf118); Alcoholism (C3orf59); Attention Deficit Disorder (C12orf28); Psychomotor Performance (C11orf73) and Erectile Dysfunction (C9orf3). These ORFs may offer novel opportunities for diagnosis and therapy of various diseases.

\section{Clinical relevance}

The NCBI ClinVar tool identified 21 ORFs in the dark matter ORFs, which are highly clinically relevant in diseases of the eyes and skin, inherited genetic disorders, developmental disorders and the neurodegenerative diseases. For example,

Truncating mutations in C2ORF71 were identified in three unrelated families causing autosomal-recessive retinitis pigmentosa (RP). Clinically, patients with mutations in C2ORF71 show signs of typical RP. ${ }^{79}$ The C2orf71 mutations were also associated with developmental delay. ${ }^{80}$ The C2orf71 encodes a transmembrane photoreceptor. Another ORF, the C8orf37| small talk protein is also highly correlated with RP and Cone-rod dystrophy (CRD). These authors showed by Immunohistochemical studies that the C8orf37 protein is localized at the base of the primary cilium of human retinal pigment epithelium cells and at the base of connecting cilia of mouse photoreceptors. ${ }^{81}$ The C8orf37 protein encodes a ciliary photoreceptor. Homozygous truncating mutation and missense mutation in C12orf57 | likely ortholog of mouse gene rich cluster was shown to be involved in the pathogenesis of a clinically distinct autosomal-recessive syndrome form of colobomatous microphthalmia, a developmental eye disorder. ${ }^{82}$ The $\mathrm{C} 12$ orf57 protein encodes a novel uncharacterized protein.

The C4orf26 loss of function mutation was associated with of recessive hypo mineralized amelogenesis imperfecta (AI). This is a disease in which the formation of tooth enamel fails. ${ }^{83}$ The C4orf26 encodes a putative extracellular matrix acidic phosphoprotein expressed in the enamel organ. Joubert syndrome (JBTS) is an autosomal-recessive disorder characterized by a distinctive midhindbrain malformation, developmental delay with hypotonia, ocular-motor apraxia, and breathing abnormalities. Rare compoundheterozygous mutations in C5orf42 have been shown to cause JBTS in French Canadian individuals. ${ }^{84}$ The C5orf42 encodes a novel uncharacterized, transmembrane coiled-coiled protein.

An expansion of a non coding GGGGCC hexanucleotide repeat in the gene C9orf72 was shown to be strongly associated with autosomaldominant frontotemporal dementia (FTD) and amyotrophic lateral sclerosis (ALS), genetically linked to chromosome 9p21. ${ }^{85,86}$ The gene C9orf72 encodes for a GDP/GTP exchange factor (GEF). Mutations in C10orf11 | Fasting-induced gene protein, a melanocyte-differentiation gene, has been shown to cause autosomal-recessive albinism, which is a hypopigmentation disorder. ${ }^{87}$ The $\mathrm{C} 10$ orf11 encodes for a Leucinerich repeat, small nuclear Ribonucleoprotein A. Missense mutations in the $\mathrm{C} 15$ orf 41 gene were implicated in congenital dyserythropoietic anemias. ${ }^{88}$ The C15orf41 protein with two predicted helix-turnhelix domains encodes a novel restriction endonuclease, bearing the Holliday junction resolvase family signature. An $11 \mathrm{bp}$ deletion in the C19orf12 | spastic paraplegia 43 (autosomal recessive) has been shown to cause neurodegeneration with brain iron accumulation. ${ }^{89}$ This gene encodes a Mitochondrial Transmembrane Iron binding protein.

These results demonstrate that the uncharacterized components of the human genome provide valuable clues to understanding biology, genetics of diseases, novel diagnostic and therapeutic opportunities.

\section{Conclusion}

Results presented in this study shed light on the uncharacterized ORFs, the Dark Matter of the human proteome. Verification of motifs and domains, expression in body fluids and association with diverse disease phenotypes offer novel therapeutic and biomarkers potential for these ORFs. Protein classes encompassing channel proteins, enzymes, receptors, secreted molecules, transcription factors and transporters were identified. The atlas of the 800 novel proteins developed in this study provides an attractive starting point for accelerated drug discovery and diagnostic markers development for major diseases.

\section{Contributions}

$\mathrm{RN}$ was responsible for conceiving, data mining, batch analysis and overall execution of the project. Data validation and visualization was performed by APD. MJC was responsible for Motifs and domains analysis. $\mathrm{PB}$ was responsible for mining the COSMIC and the NextBio databases. SH was responsible for mining the HPA, Roche Mutome and HPRD databases. 


\section{Acknowledgements}

We thank Dr. Stein, GeneCards team for generous permission to use the GeneALaCart tool, Dr. Libby Montague, Kolker Laboratory, MOPED Team for the batch analysis of the ORFs; the NextBio Meta analysis tool for permission to use the dataset; the CanSar, cBioPortal, the TCGA and COSMIC for the mutations dataset, the Human Protein Atlas, Human Protein Reference Database and DAVID functional annotations tools for various datasets. We especially thank the human proteome map and the proteomes $\mathrm{dB}$ (Dr. Mathias Wilhelm) for the protein expression datasets. This work was supported in part by the Genomics of Cancer Fund, Florida Atlantic University Foundation. We thank Jeanine Narayanan for editorial assistance.

\section{Conflict of interest}

The author declares no conflict of interest.

\section{References}

1. Blaxter M. Genetics. Revealing the dark matter of the genome. Science. 2010;330(6012):1758-1759.

2. Hopkins AL, Groom CR. The druggable genome. Nat Rev Drug Discov. 2002;1(9):727-730.

3. Andersen MR, Almen MS, Schioth HB. Trends in the exploitation of novel drug targets. Nat Rev Drug Discov. 2011;10(8):579-590.

4. Nadzirin N, Raih MF. Proteins of Unknown Function in the Protein Data Bank (PDB): An Inventory of True Uncharacterized Proteins and Computational Tools for Their Analysis. Int J Mol Sci. 2012;13(10):12761-12772.

5. Martin L, Chang HY. Uncovering the role of genomic "dark matter" in human disease. J Clin Invest. 2012;122(5):1589-1595.

6. Mullard A. Drug makers and NIH team up to find and validate targets. Nat Rev Drug Discov. 2014;13(4):241-243.

7. Narayanan R. Ebola-associated genes in the human genome. Implications for novel targets. MOJ Proteomics Bioinform. 2014;1(5):1-7.

8. Narayanan R. Neurodegenerative diseases: Phenome to genome analysis. MOJ Proteomics Bioinform. 2014;1(6):1-10.

9. Narayanan R. Phenome-Genome association studies of pancreatic cancer: New targets for therapy and diagnosis. Cancer Genomics Proteomics. 2015;12(1):9-19.

10. Delgado AP, Brandao P, Chapado M, et al. Open Reading Frames Associated with Cancer in the Dark Matter of the Human Genome. Cancer Genomics Proteomics. 2014;11(4):201-213.

11. Delgado AP, Brandao P, Narayanan R. Diabetes Associated Genes from the Dark Matter of the Human Proteome. MOJ Proteomics Bioinform. 2014;1(4):1-8

12. Zhang Y, De S, Garner JR, et al. Systematic analysis, comparison, and integration of disease based human genetic association data and mouse genetic phenotypic information. BMC Med Genomics. 2010;3:1.

13. Rappaport N, Nativ N, Stelzer G, et al. MalaCards: an integrated compendium for diseases and their annotation. Database (Oxford). 2013;2013:bat018

14. Delgado AP, Brandao P, Hamid S, et al. Mining the Dark Matter of the Cancer Proteome for novel biomarkers. Current Cancer Therapy Reviews. 2013;9(4):265-277.

15. Delgado A, Hamid S, Brandao $\mathrm{P}$, et al. A novel transmembrane glycoprotein cancer biomarker present in the $\mathrm{x}$ chromosome. Cancer Genomics Proteomics. 2014;11(2):81-92.
16. Becker $\mathrm{KG}$, Barnes $\mathrm{KC}$, Bright $\mathrm{TJ}$, et al. The genetic association database. Nat Genet. 2004;36(5):431-432.

17. Karolchik D, Barber GP, Casper J, et al. The UCSC Genome Browser database: 2014 update. Nucleic Acids Res. 2013;42(Database issue):D764-770.

18. Flicek P, Amode MR, Barrell D, et al. Ensembl 2014. Nucleic Acids Res 2014;2(Database issue):D749-755.

19. Mieg DT, Mieg JT. AceView: a comprehensive cDNA-supported gene and transcripts annotation. Genome Biol. 2006;7(Suppl 1):S12.1-14.

20. Forbes SA, Bindal N, Bamford S, et al. COSMIC: mining complete cancer genomes in the Catalogue of Somatic Mutations in Cancer. Nucleic Acids Res. 2011;39(Database issue):D945-D950.

21. Brown HMD, Bulusu KC, Patel M, et al. canSAR: an integrated cancer public translational research and drug discovery resource. Nucleic Acids Res. 2012;40(Database issue):D947-956.

22. Cerami E, Gao J, Dogrusoz U, et al. The cBio cancer genomics portal an open platform for exploring multidimensional cancer genomics data. Cancer Discov. 2012;2(5):401-404.

23. International Cancer Genome C, Hudson TJ, Anderson W, et al. International network of cancer genome projects. Nature. 2010;464(7291):993-998.

24. Kuntzer J, Maisel D, Lenhof HP, et al. The Roche Cancer Genome Database 2.0. BMC Med Genomics. 2011;4:43.

25. Ramos EM, Hoffman D, Junkins HA, et al. Phenotype-Genotype Integrator (PheGenI): synthesizing genome-wide association study (GWAS) data with existing genomic resources. Eur J Hum Genet. 2014;22(1):144-147.

26. Consortium GT. The Genotype-Tissue Expression (GTEx) project. Nat Genet. 2013;45(6):580-585.

27. Velculescu VE, Zhang L, Vogelstein B, et al. Serial analysis of gene expression. Science. 1995;270(5235):484-487.

28. Strausberg RL. The Cancer Genome Anatomy Project: new resources for reading the molecular signatures of cancer. J Pathol. 2001;195(1):31-40.

29. Rhodes DR, Sundaram SK, Mahavisno V, et al. Oncomine 3.0: genes, pathways, and networks in a collection of 18,000 cancer gene expression profiles. Neoplasia. 2007;9(2):166-180.

30. Parkinson H, Sarkans U, Shojatalab M, et al. ArrayExpress-a public repository for microarray gene expression data at the EBI. Nucleic Acids Res. 2005;31(1):68-71.

31. Liu F, White JA, Antonescu C, et al. GCOD- GeneChip Oncology Database. BMC Bioinformatics. 2011;12:46.

32. UniProt C. Update on activities at the Universal Protein Resource (UniProt) in 2013. Nucleic Acids Res. 2013;41(Database issue):D43D47.

33. Artimo P, Jonnalagedda M, Arnold K, et al. ExPASy: SIB bioinformatics resource portal. Nucleic Acids Res. 2012;40(Web Server issue):W597W603.

34. Nair R, Rost B. LOCnet and LOCtarget: sub-cellular localization for structural genomics targets. Nucleic Acids Res. 2004;32(Web Server issue):W517-W521.

35. Cong Q, Grishin NV. MESSA: MEta-Server for protein Sequence Analysis. BMC Biol. 2012;10:82.

36. Zhang Y. I-TASSER server for protein 3D structure prediction. BMC bioinformatics. 2008;9:40.

37. Bauer AM, Zheng C, Chitsaz F, et al. CDD: conserved domains and protein three-dimensional structure. Nucleic Acids Res. 2013;41(Database issue):D348-D352. 
38. Finn RD, Bateman A, Clements J, et al. Pfam: the protein families database. Nucleic Acids Res. 2014;40(Database issue):D290-D301.

39. Servant F, Bru C, Carrere S, et al. ProDom: automated clustering of homologous domains. Brief Bioinform. 2002;3(3):246-251.

40. Hunter S, Apweiler R, Attwood TK, et al. InterPro: the integrative protein signature database. Nucleic Acids Res. 2009;37(Database issue):D211D215.

41. Finn RD, Clements J, Eddy SR. HMMER web server: interactive sequence similarity searching. Nucleic Acids Res. 2011;39(Web Server issue):W29-W37.

42. Petersen TN, Brunak S, von Heijne G, et al. SignalP 4.0: discriminating signal peptides from transmembrane regions. Nat Methods. 2011;8(10):785-786.

43. Dinkel H, Van Roey K, Michael S, et al. The eukaryotic linear motif resource ELM:10 years and counting. Nucleic Acids Res. 2014;42(Database issue):D259-D266.

44. Uhlen M, Oksvold P, Fagerberg L, et al. Towards a knowledge-based Human Protein Atlas. Nat Biotechnol. 2010;28(12):1248-1250.

45. Kolker E, Higdon R, Haynes W, et al. MOPED: Model Organism Protein Expression Database. Nucleic Acids Res. 2012;40(Database issue):D1093-D1099.

46. Mathivanan S, Ahmed M, Ahn NG, et al. Human Proteinpedia enables sharing of human protein data. Nat Biotechnol. 2008;26(2):164-167.

47. Kim MS, Pinto SM, Getnet D, et al. A draft map of the human proteome. Nature. 2014;509(7502):575-581.

48. Maruyama Y, Kawamura Y, Nishikawa T, et al. HGPD: Human Gene and Protein Database, 2012 update. Nucleic Acids Res. 2012;40(Database issue):D924-D929.

49. Wilhelm M, Schlegl J, Hahne H, et al. Mass-spectrometry-based draft of the human proteome. Nature. 2014;509(7502):582-587.

50. Safran M, Dalah I, Alexander J, et al. GeneCards Version 3:the human gene integrator. Database (Oxford). 2010;2010:baq020.

51. Frezal J. Genatlas database, genes and development defects. C R Acad Sci III. 1998;321(10):805-817.

52. Kupershmidt I, Su QJ, Grewal A, et al. Ontology-based meta-analysis of global collections of high-throughput public data. PLoS One. 2010:5(9):e13066

53. Ashburner M, Ball CA, Blake JA, et al. Gene ontology:tool for the unification of biology. The Gene Ontology Consortium. Nat Genet. 2000;25(1):25-29.

54. Landrum MJ, Lee JM, Riley GR, et al. ClinVar: public archive of relationships among sequence variation and human phenotype. Nucleic Acids Res. 2014;42(Database issue):D980-D985.

55. Franceschini A, Szklarczyk D, Frankild S, et al. STRING v9.1:proteinprotein interaction networks, with increased coverage and integration. Nucleic acids research. Nucleic Acids Res. 2013;41(Database issue):D808-D815.

56. Stark C, Breitkreutz BJ, Aryamontri AC, et al. The BioGRID Interaction Database:2011 update. Nucleic Acids Res. 2011;39(Database issue):D698-D704.

57. Orchard S, Ammari M, Aranda B, et al. The MIntAct project-IntAct as a common curation platform for 11 molecular interaction databases. Nucleic Acids Res. 2013;42(Database issue):D358-363.

58. Huang DW, Sherman BT, Lempicki RA. Systematic and integrative analysis of large gene lists using DAVID bioinformatics resources. Nat Protoc. 2008;4(1):44-57.
59. Weiler T, Du Q, Krokhin O, et al. The identification and characterization of a novel protein, c19orf10, in the synovium. Arthritis Res Ther. 2007;9(2):R30.

60. Sunagozaka H, Honda M, Yamashita T, et al. Identification of a secretory protein c19orf10 activated in hepatocellular carcinoma. Int J Cancer. 2011;129(7):1576-1585.

61. Buning C, Genschel J, Weltrich R, et al. The interleukin-25 gene located in the inflammatory bowel disease (IBD) 4 region: no association with inflammatory bowel disease. Eur J Immunogenet. 2003;30(5):329-333.

62. Benusiglio PR, Pharoah PD, Smith PL, et al. HapMap-based study of the 17q21 ERBB2 amplicon in susceptibility to breast cancer. Br J Cancer. 2006;95(12):1689-1695.

63. Mavaddat N, Dunning AM, Ponder BA, et al. Common genetic variation in candidate genes and susceptibility to subtypes of breast cancer. Cancer Epidemiol Biomarkers Prev. 2009;18(1):255-259.

64. Liang L, Morar N, Dixon AL, et al. A cross-platform analysis of 14,177 expression quantitative trait loci derived from lymphoblastoid cell lines. Genome Res. 2013;23(4):716-726.

65. Fellay J, Ge D, Shianna KV, et al. Common genetic variation and the control of HIV-1 in humans. PLoS Genet. 2009;5(12):e1000791.

66. Barcellos LF, May SL, Ramsay PP, et al. High-density SNP screening of the major histocompatibility complex in systemic lupus erythematosus demonstrates strong evidence for independent susceptibility regions. PLoS Genet. 2009;5(10):e1000696.

67. Johnson AD, Yanek LR, Chen MH, et al. Genome-wide meta-analyses identifies seven loci associated with platelet aggregation in response to agonists. Nat Genet. 2010;42(7):608-613.

68. Uhl GR, Liu QR, Drgon T, et al. Molecular genetics of successful smoking cessation: convergent genome-wide association study results. Arch Gen Psychiatry. 2008;65(6):683-693.

69. Rose JE, Behm FM, Drgon T, et al. Personalized smoking cessation: interactions between nicotine dose, dependence and quit-success genotype score. Mol Med. 2010;16(7-8):247-253.

70. Gerards M, Sluiter W, van den Bosch BJ, et al. Defective complex I assembly due to $\mathrm{C} 20$ orf7 mutations as a new cause of Leigh syndrome. $J$ Med Genet. 2010;47(8):507-512.

71. Sugiana C, Pagliarini DJ, McKenzie M, et al. Mutation of C20orf7 disrupts complex I assembly and causes lethal neonatal mitochondrial disease. Am J Hum Genet. 2008;83(4):468-478.

72. Mulder NJ, Apweiler R, Attwood TK, et al. InterPro: an integrated documentation resource for protein families, domains and functional sites. Brief Bioinform. 2002;3(3):225-235.

73. Punta M, Coggill PC, Eberhardt RY, et al. The Pfam protein families database. Nucleic Acids Res. 2012;40(Database issue):D290-D301.

74. Kent WJ, Sugnet CW, Furey TS, et al. The human genome browser at UCSC. Genome Res. 2002;12(6):996-1006.

75. Zhang B, Wang J, Wang X, et al. Proteogenomic characterization of human colon and rectal cancer. Nature. 2014;513(7518):382-387.

76. Narayanan R, Van De Ven WJM. Transcriptome and Proteome Analysis: A Perspective on Correlation. MOJ Proteomics Bioinform. 2014;1(5):12 .

77. Hauptman N, Glavac D. MicroRNAs and long non-coding RNAs: prospects in diagnostics and therapy of cancer. Radiol Oncol. 2013;47(4):311-318.

78. Costa PM, Pedroso de Lima MC. MicroRNAs as Molecular Targets for Cancer Therapy: On the Modulation of MicroRNA Expression. Pharmaceuticals (Basel). 2013;6(10):1195-1220. 
79. Collin RW, Safieh C, Littink KW, et al. Mutations in C2ORF71 cause autosomal-recessive retinitis pigmentosa. Am J Hum Genet. 2010;86(5):783-788

80. Kaminsky EB, Kaul V, Paschall J, et al. An evidence-based approach to establish the functional and clinical significance of copy number variants in intellectual and developmental disabilities. Genet Med. 2011;13(9):777-784.

81. Cuzcano AE, Neveling $\mathrm{K}$, Kohl S, et al. Mutations in C8orf37, encoding a ciliary protein, are associated with autosomal-recessive retinal dystrophies with early macular involvement. Am J Hum Genet. 2012;90(1):102-119.

82. Zahrani F, Aldahmesh MA, Alshammari MJ, et al. Mutations in c12orf57 cause a syndromic form of colobomatous microphthalmia. Am J Hum Genet. 2013;92(3):387-391.

83. Parry DA, Brookes SJ, Logan CV, et al. Mutations in C4orf26, encoding a peptide with in vitro hydroxyapatite crystal nucleation and growth activity, cause amelogenesis imperfecta. Am J Hum Genet. 2012;91(3):565-571

84. Srour M, Schwartzentruber J, Hamdan FF, et al. Mutations in C5ORF42 cause Joubert syndrome in the French Canadian population. Am J Hum Genet. 2012;90(4):693-700.
85. Hernandez MD, Mackenzie IR, Boeve BF, et al. Expanded GGGGCC hexanucleotide repeat in noncoding region of C9ORF72 causes chromosome 9p-linked FTD and ALS. Neuron. 2011;72(2):245-256.

86. Van der Zee J, Gijselinck I, Dillen L, et al. A pan-European study of the C9orf72 repeat associated with FTLD:geographic prevalence, genomic instability, and intermediate repeats. Hum Mutat. 2013;34(2):363-373.

87. Gronskov K, Dooley CM, Ostergaard E, et al. Mutations in c10orf11, a melanocyte-differentiation gene, cause autosomal-recessive albinism. Am J Hum Genet. 2013;92(3):415-421.

88. Babbs C, Roberts NA, Sanchez-Pulido L, et al. Homozygous mutations in a predicted endonuclease are a novel cause of congenita dyserythropoietic anemia type I. Haematologica. 2013;98(9):13831387.

89. Hartig MB, Iuso A, Haack T, et al. Absence of an orphan mitochondrial protein, c19orf12, causes a distinct clinical subtype of neurodegeneration with brain iron accumulation. Am J Hum Genet. 2011;89(4):543-550. 